\title{
Oscillations and running waves observed in sunspots
}

\author{
III. Multilayer study \\ E. B. Christopoulou ${ }^{1}$, A. A. Georgakilas ${ }^{2}$, and S. Koutchmy ${ }^{3}$ \\ 1 Electronics Laboratory, University of Patras, Patras 26110, Greece \\ e-mail: echris@physics.upatras.gr \\ 2 Solar Astronomy, California Institute of Technology, Pasadena, CA 91125, USA \\ e-mail: ageorg@caltech.edu \\ 3 Institut d'Astrophysique de Paris, CNRS, 98 bis boulevard Arago, 75014 Paris, France \\ e-mail: koutchmy@iap.fr
}

Received 12 April 2001 / Accepted 28 May 2001

\begin{abstract}
We continue our study of waves and oscillations observed in sunspots using an improved method for enhancing the waves, giving the opportunity to identify them and determine their properties in far $\mathrm{H} \alpha$ wings. We found that the running penumbral waves are observable at least up to the formation height of the $\mathrm{H} \alpha \pm 0.5 \AA$ line, but not in the $\mathrm{H} \alpha \pm 0.75 \AA$ or the $\mathrm{Fe} \mathrm{I} \pm 0.12 \AA$. We found a time lag between the waves in the blue and the red wing of the $\mathrm{H} \alpha$ line corresponding to a phase shift of $180^{\circ}$, that indicates a pure Doppler shift of the line. There is a lag in the propagation of the waves seen at $\mathrm{H} \alpha$ center and at $\mathrm{H} \alpha$ wings. Also there is a lag in the variation of the umbral oscillations as they are observed from lower to higher atmospheric layers. The correlation between umbral oscillations at various atmospheric heights and running penumbral waves strongly indicates that the latter are excited by photospheric umbral oscillations and not the chromospheric ones. We found a new category of photospheric waves that originate at approximately 0.7 of the distance between the umbra and the penumbra boundary and propagate beyond the outer penumbra boundary with a velocity of the order of 3-4 $\mathrm{km} \mathrm{s}^{-1}$. Further, we found $3 \mathrm{~min}$ penumbral oscillations apparent in the inner penumbra at lower chromospheric layers (far $\mathrm{H} \alpha$ wings).
\end{abstract}

Key words. Sun: sunspots - Sun: oscillations

\section{Introduction}

Sunspots show oscillatory behavior, identifiable as intensity and velocity variations in photospheric and chromospheric lines. In the photosphere two types of characteristic oscillations are observed in the umbra; one with period around $5 \mathrm{~min}$ (response to forcing by the $p$-mode photospheric oscillations) and one with period around $3 \mathrm{~min}$ (believed to be related to resonant modes of the spot itself) (Lites \& Thomas 1985). Observations of the 5-min oscillations in purely umbral lines, like the $\mathrm{TiO}$, establish the umbral origin of this oscillation mode (see for instance Soltau et al. 1976). According to Lites (1992) the oscillatory motions measured in spectral lines formed in the lower photosphere of umbrae show a dominant power peak centered around 5-min periods. In the chromospheric lay-

Send offprint requests to: A. A. Georgakilas, e-mail: ageorg@CALTECH. edu

* Based on observations performed on the NSO/SPO Richard B. Dunn Solar Telescope (DST) ers 3 min standing oscillations are dominant in the umbra, while running 5 min waves are dominant in the penumbra and superpenumbra. The 5-min umbral oscillations have either very low amplitudes or they are not present at all (Lites 1992). Running penumbral waves (RPWs) were first observed in $\mathrm{H} \alpha$ by Zirin \& Stein (1972) and independently by Giovanelli $(1972,1974)$. Since their discovery researchers have tried to determine their nature, how deeply seated they are, as well as their relation to other phenomena such as umbral oscillations and $p$-mode photospheric oscillations. A recent comprehensive review of oscillatory phenomena related to sunspots is given by Bogdan (2000).

In the first paper of this series (Christopoulou et al. 2000, Paper I from now on), we studied oscillations and waves related to sunspots in the chromosphere. We found that standing umbral oscillations are dominant in the umbra and inner penumbra with a frequency of about $6.5 \mathrm{mHz}$, running waves propagate in the penumbra and inner superpenumbra with constant velocity 

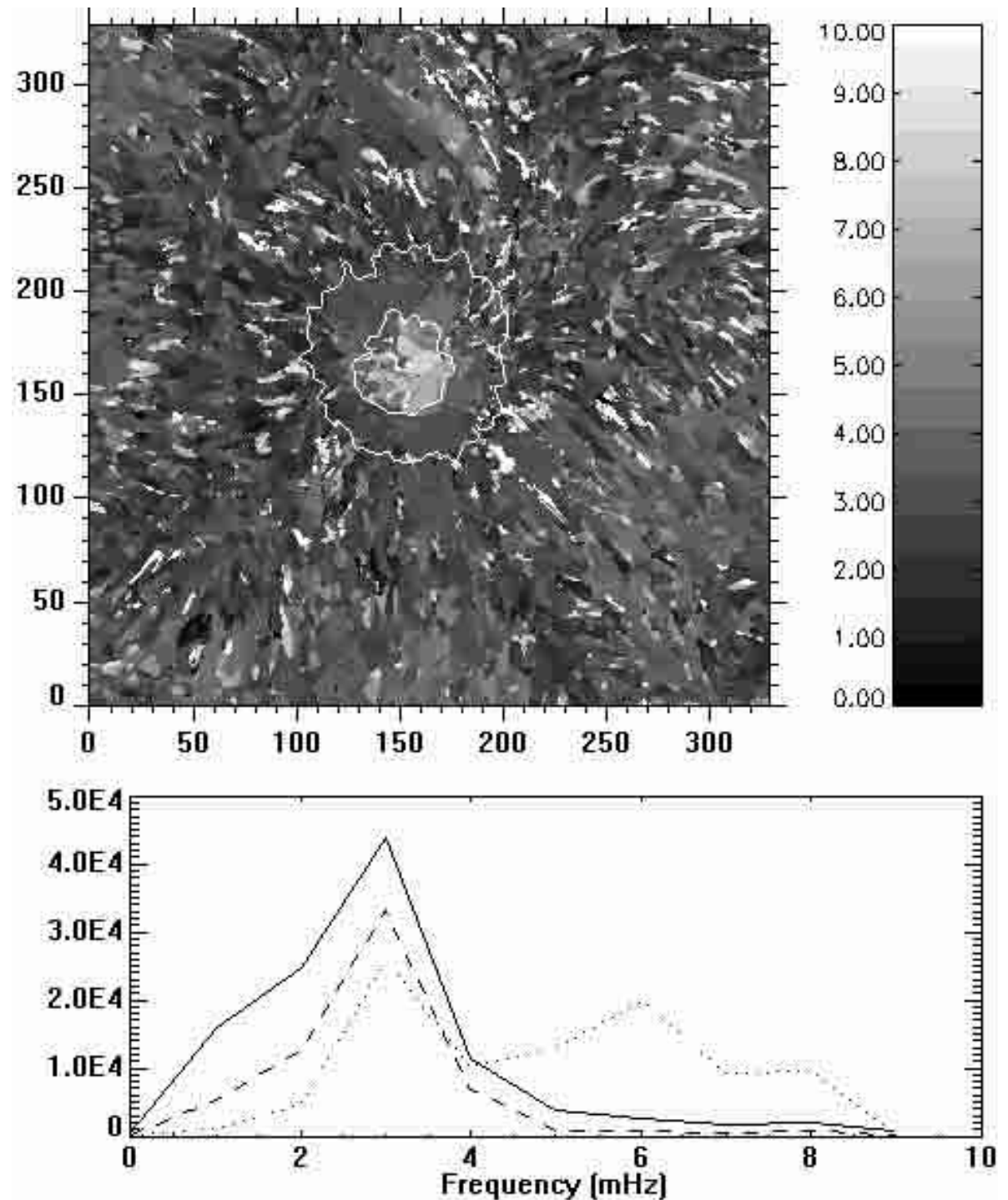

Fig. 1. Map of "frequencies of maximum spectral power" computed from $\mathrm{H} \alpha-0.35 \AA$ images, using a 128-point FFT. In order to produce the map we computed the power spectrum for each pixel of the map and selected the maximum power frequency as the most representative oscillating frequency for this pixel. Contours delineate the umbra and the penumbra as they appear in Fe I (5576.099) $\pm 0.12 \AA$ average map. The histogram below the map shows the frequency distribution of all pixels (solid line) and of pixels related to the umbra(dotted line) and the penumbra (dashed line). In order to make the three distributions comparable we have multiplied the distribution of the pixels related to the umbra and the penumbra by a factor of 50 and 10 respectively (see text for further details). The intensity bar on the right of the image, scales brightness to frequency values in $\mathrm{mHz}$.

around $13 \mathrm{~km} \mathrm{~s}^{-1}$ (frequency around $3 \mathrm{mHz}$ ), and oscillations in the penumbra-superpenumbra boundary and outwards with frequency around $2 \mathrm{mHz}$ or lower. Further, we concluded that there is not a clear relationship between umbral oscillations and running penumbral waves. If RPWs are generated by umbral oscillations or by the same cause with umbral oscillations, then a mechanism which will explain the different period is necessary.

In the second paper of the series (Georgakilas et al. 2000 ), we studied waves related to sunspots in the photosphere. We found inward slow propagating waves in the photospheric penumbra and outward propagating waves in the area around the sunspot. The phase velocity of the waves is near $0.5 \mathrm{~km} \mathrm{~s}^{-1}$ in both cases and their horizontal wavelength about $2500 \mathrm{~km}$. The waves are probably related to the subphotospheric layer large-scale convection.

Brisken \& Zirin (1997) found that there was a time lag in the observation of the waves at a given point between $\mathrm{H} \alpha$ and $\mathrm{H} \alpha \pm 0.6 \AA$. Based on this, they proposed that the waves are due to the expansion of a spherical wavefront from a point about $15 \mathrm{Mm}$ below the surface. On the other hand, Moore (1981) from the analysis of $\mathrm{H} \alpha$ spectral observations found that the entire line is shifted in these oscillations; the two wings of the line are simultaneously shifted towards the same direction by the same 

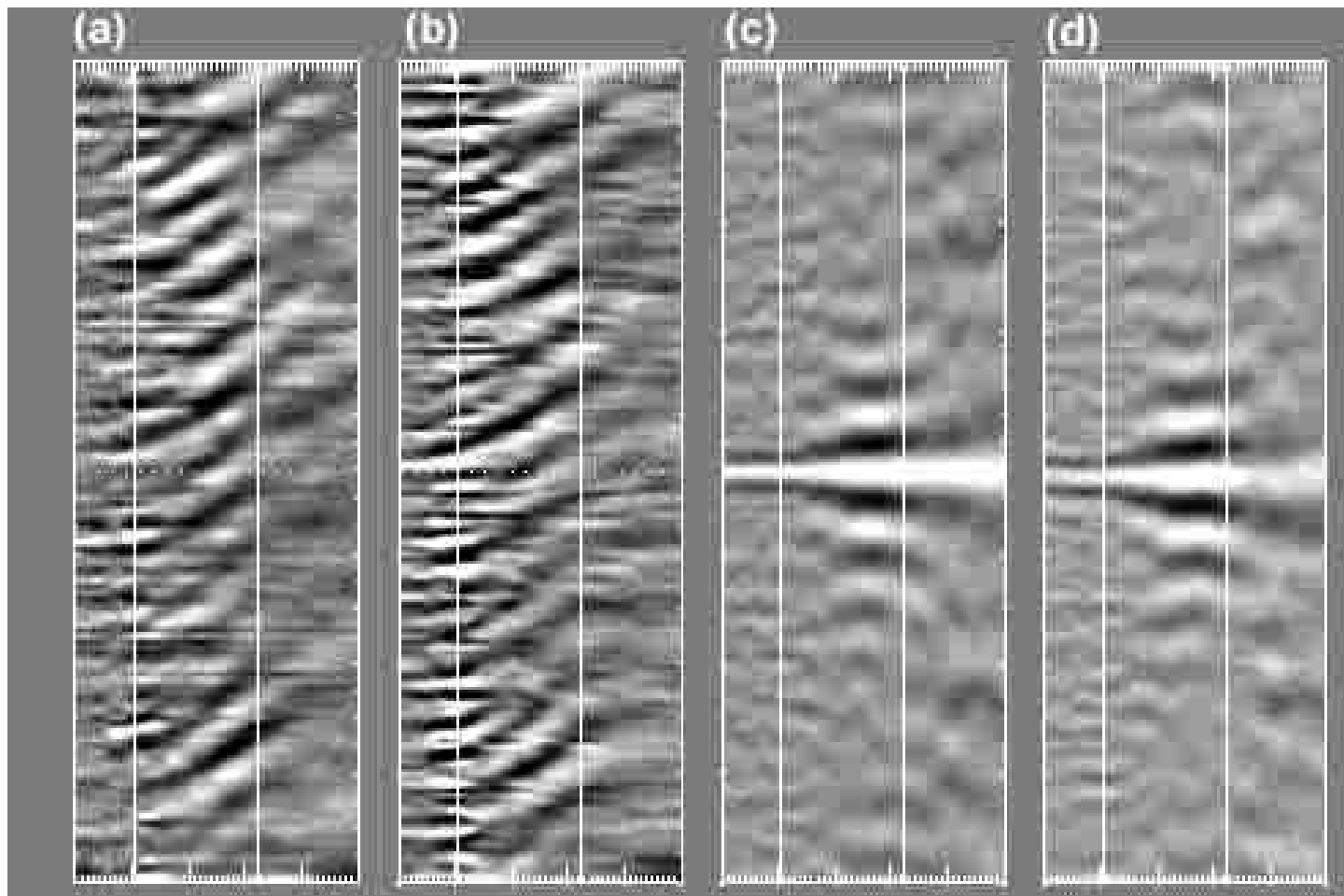

Fig. 2. "Space/time slice" images in $\mathrm{H} \alpha$ center (a) and $\mathrm{H} \alpha-0.35 \AA$ (b), the "autocorrelogram" of the H $\alpha$ center image (c) and the "correlogram" of the two images (d), showing a correlation of the umbral oscillations and RPWs in the two wavelengths. See text for the meaning of the "correlogram" and how it is computed. Tick marks in the $x$-axis correspond to 0.26 " and tick marks in the $y$-axis to $140 \mathrm{~s}$.

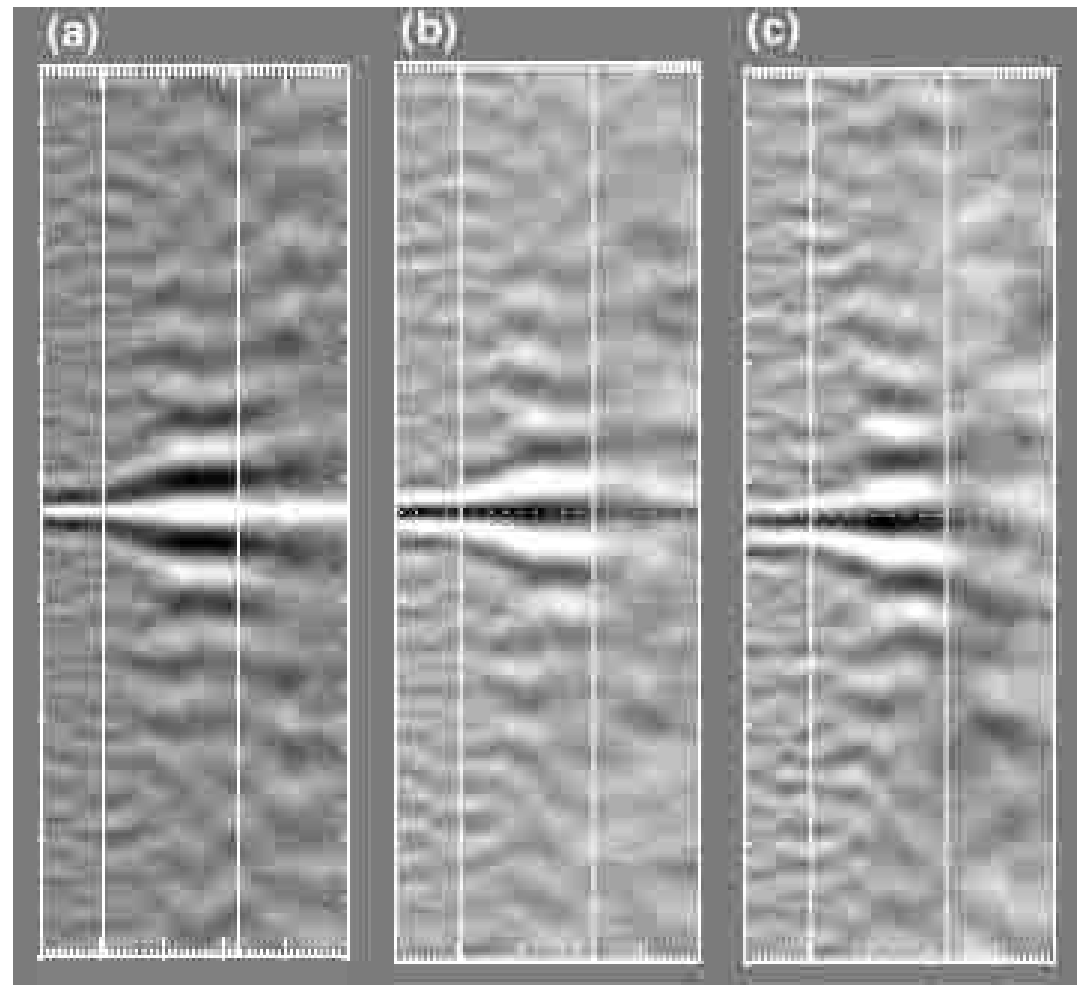

Fig. 3. "Autocorrelogram" of the $\mathrm{H} \alpha-0.35 \AA$ "time slice image" (a), "correlogram" between $\mathrm{H} \alpha-0.35 \AA$ and Doppler velocity (b) and "correlogram" between $\mathrm{H} \alpha$ center and Doppler velocity (c) (see Fig. 2). Tick marks in the $x$-axis correspond to 0.26 "and tick marks in the $y$-axis to $140 \mathrm{~s}$. 


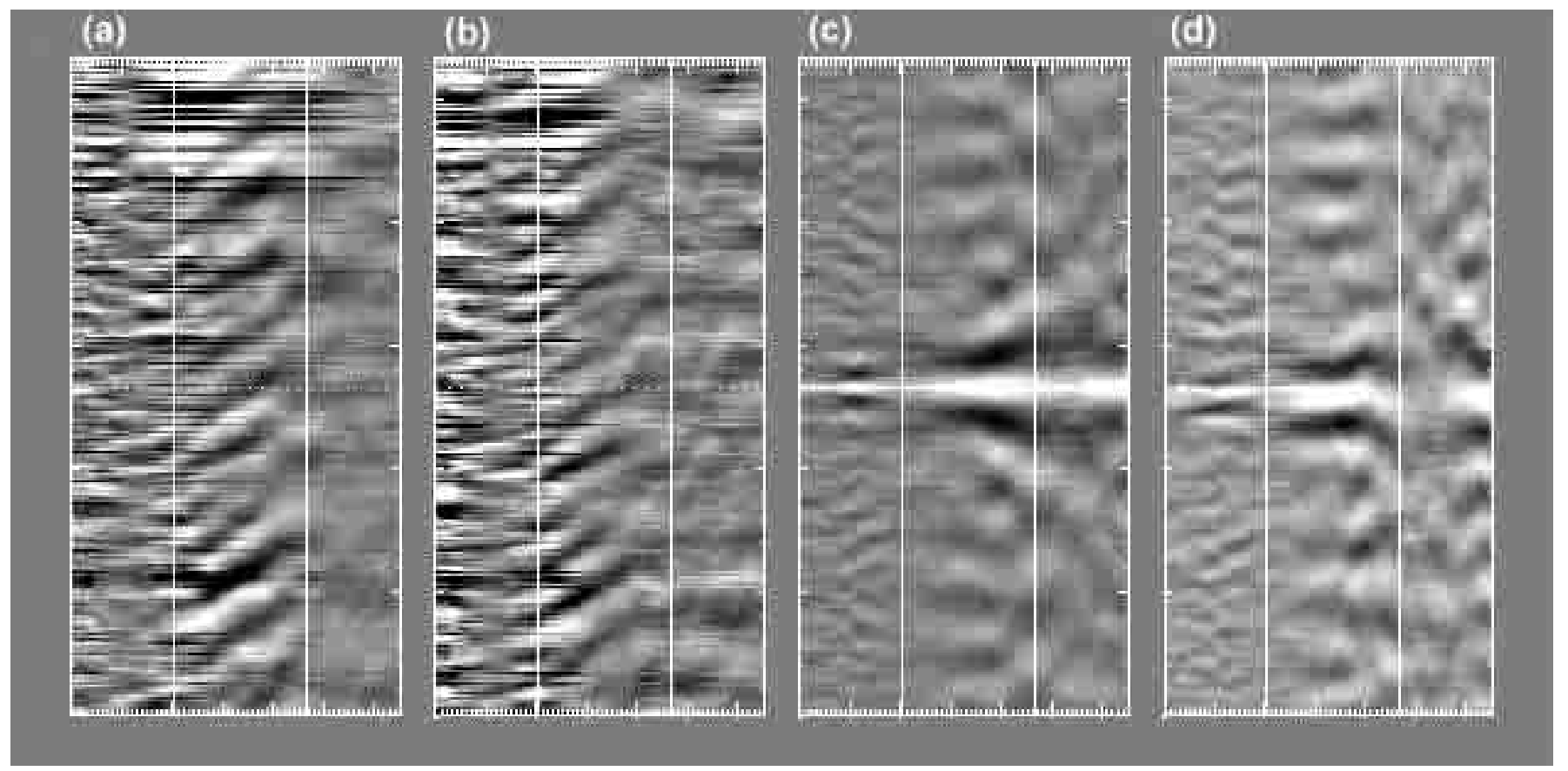

Fig. 4. "Space/time slice" images in $\mathrm{H} \alpha$ (a) and $\mathrm{H} \alpha-0.5 \AA$ (b), the "autocorrelogram" of the H $\alpha$ image (c) and the "correlogram" of the two images (d), showing a correlation of the umbral oscillations and RPWs in the two wavelengths (see Fig. 2). Tick marks in the $x$-axis correspond to $0.26^{\prime \prime}$ and tick marks in the $y$-axis to $120 \mathrm{~s}$.

amount. This led him to conclude that the full thickness of the penumbral chromosphere moves up and down in the waves.

In this paper we focus our research on the comparison of running waves and umbral oscillations in different layers of the solar atmosphere as well as their relation.

\section{Observations and image processing}

Observations were obtained at the R. B. Dunn telescope of the Sacramento Peak Observatory with a 512 by 512 pixel CCD camera and the $U B F$ filter. The pixel spatial resolution was $0.26^{\prime \prime}$. A large isolated sunspot was observed at N14.7, E26.0 on August 15, 1997. We obtained three sequences of observations one after the other. Table I summarizes the observations; further details can be found in Papers I and II. Note that the precision of the $U B F$ filter is of the order of $1 \mathrm{~m} \AA$, while the FWHM is about $240 \mathrm{~m} \AA$ near $\mathrm{H} \alpha$ and about $120 \mathrm{m \AA}$ near Fe I (5576.099). We computed Dopplergrams in $\mathrm{H} \alpha \pm 0.35, \pm 0.5, \pm 0.75 \AA$ as well as in Fe $\mathrm{I} \pm 0.12 \AA$ by red blue wing subtraction. We used the Doppler images as qualitative maps of the line of sight velocity.

In order to remove the sharp intensity gradient between the umbra and the penumbra and to enhance time varying phenomena in intensity images, we subtracted from each image the "running local average", computed over a number of images before and after it. The "running local average" covers a few periods of the waves in order to produce a background free of waves. Details about the method can be found in Paper I and references there in. The method makes the waves much more noticeable without introducing artifacts (see Fig. 1 of Paper I). We should note that although the subtraction of the "running local average" enhances waves with shorter periods than the integration time, it washes out waves with larger periods.

In Paper I, in order to study the properties of umbral oscillation and running waves, we took a cross section of every image of a time series, starting from the center of an oscillating element and directed outwards, at right angles to the propagating wavefronts. We created a new image with the distance from the center of the oscillating elements as one axis and time as the other (method I). In Paper II we applied an improved method to photospheric observations; we computed the average intensity along circular arcs at right angles to a line directed outwards beginning from the center of the oscillating element (method II). The line is obtained at right angles to the wavefronts to have the arcs parallel to the wavefronts. The arcs correspond to a stable angle so that their length smoothly increases with distance from the center of the spot. This is a more appropriate method since the waves expand in larger arcs as they propagate outwards. The new method gives significantly better results, much less noise and the oscillating frequency can be determined with significantly higher confidence. Further details about the methods can be found in the corresponding papers. In this paper we use method II in order to compute "space time slice" images with an integration angle of $30^{\circ}$ except in case otherwise stated.

We used an image segmentation procedure based on a histogram technique in order to determine the area of the umbra and the penumbra and compute a binary mask, setting umbra (or penumbra) pixels to one and the rest to zero (see for instance Steinegger et al. 1997). The original image was then multiplied by this mask producing a 


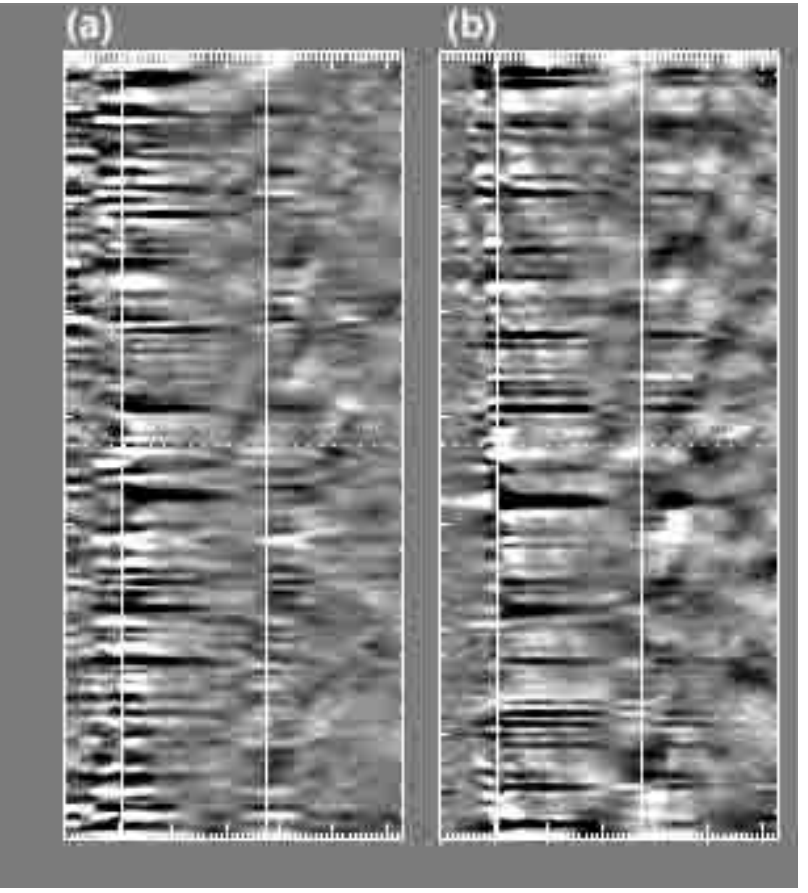

Fig. 5. "Space/time slice" images showing standing oscillations in the inner penumbra and RPWs in $\mathrm{H} \alpha-0.75 \AA$ (a) and FeI(5576.099) $-0.12 \AA$ (b). The RPWs start at about 0.7 of the distance between the umbra and the penumbra boundaries and propagate outwards in the region around the sunspot. The vertical lines mark the umbra and penumbra boundaries as derived from the Fe $\mathrm{I}-0.12 \AA$ image. Tick marks in the $x$-axis correspond to $0.26^{\prime \prime}$ and tick marks in the $y$-axis to $140 \mathrm{~s}$. Note that slow photospheric waves are washed out since from each image we have subtracted the running local average computed over a period of about $9 \mathrm{~min}$.

segmented image in which the umbra (or penumbra) pixels conserved their original intensity and the background was set to zero. Finally, we constructed maps of frequency of maximum spectral power, which we will briefly call "maximum power frequency maps". The maps were constructed by computing the power spectrum for each pixel of the map and selecting the frequency of maximum spectral power as the most representative oscillating frequency for this pixel. We compared the "maximum power frequency" maps with intensity power maps computed in selected frequency regions in order to verify that the indicated maximum power frequencies represent the most characteristic oscillating frequencies for the corresponding pixels. Figure 1 shows a "maximum power frequency map" computed from $\mathrm{H} \alpha-0.35 \AA$ images, using a 128-point fast Fourier transform (FFT). We observe that extended regions in the umbra and the penumbra and even some fibrils show a coherent behavior. We further computed the "maximum power" frequency distribution of all the pixels of the map and of the pixels related to the umbra and the penumbra. In order to compute the latter distributions we multiplied the "maximum power frequency map" with the binary mask computed from the average of the sum of the Fe $\mathrm{I} \pm 0.12 \AA$ images. We have multiplied the distribu-

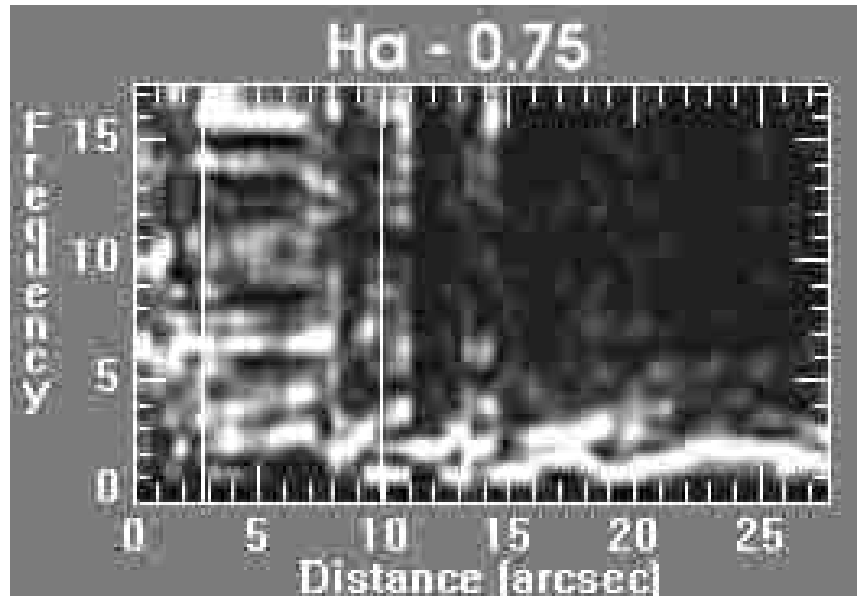

Fig. 6. Frequency/space map computed from the $\mathrm{H} \alpha-0.75 \AA$ "space/time slice image" (Fig. 5a). The vertical axis shows frequency and the horizontal distance from the center of the spot. Notice that in the inner penumbral area, except from the power at about $3 \mathrm{mHz}$, significant power is observed at about $6.25 \mathrm{mHz}$ related with standing oscillations (see Fig. 5).

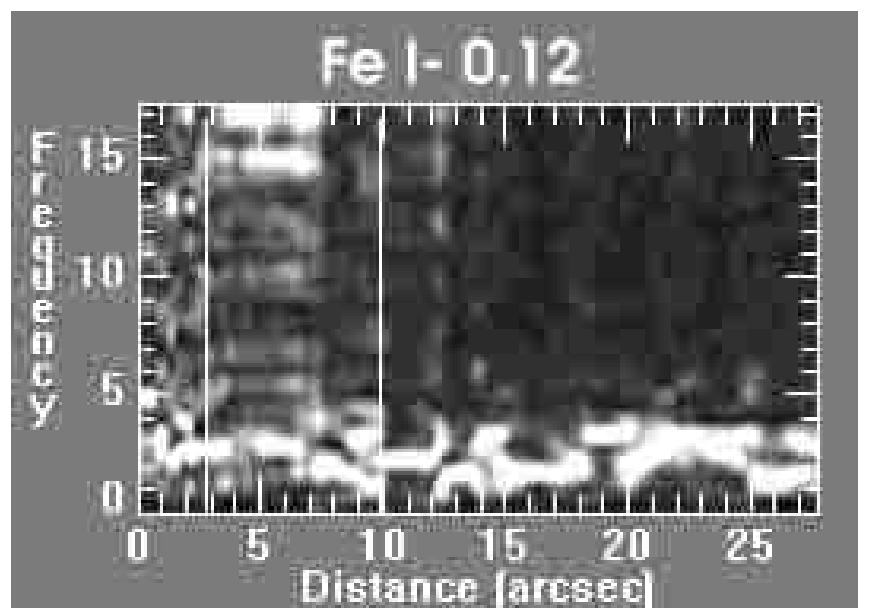

Fig. 7. Frequency/space map computed from the $\mathrm{Fe} I$ (5576.099) -0.12 $\AA$ "space/time slice image" (Fig. 5b). The vertical axis shows frequency and the horizontal distance from the center of the spot (see Fig. 6).

tion of the pixels related to the umbra and the penumbra by a factor of 50 and 10 respectively, in order to make the three disributions comparable (since the number of the umbra and the penumbra pixels is much smaller than the whole number of pixels).

\section{Results}

\subsection{Running waves and penumbral oscillations in different layers of the solar atmosphere}

In Paper I we found that running waves are best observed in about $\mathrm{H} \alpha-0.2 \AA$ and further that they are more prominent in the blue wing than the red one. Both visually and from "space/time slice images" computed using method I (see chapter 2), we could barely observe them in $\mathrm{H} \alpha \pm 0.5 \AA$ and we could not observe them in 

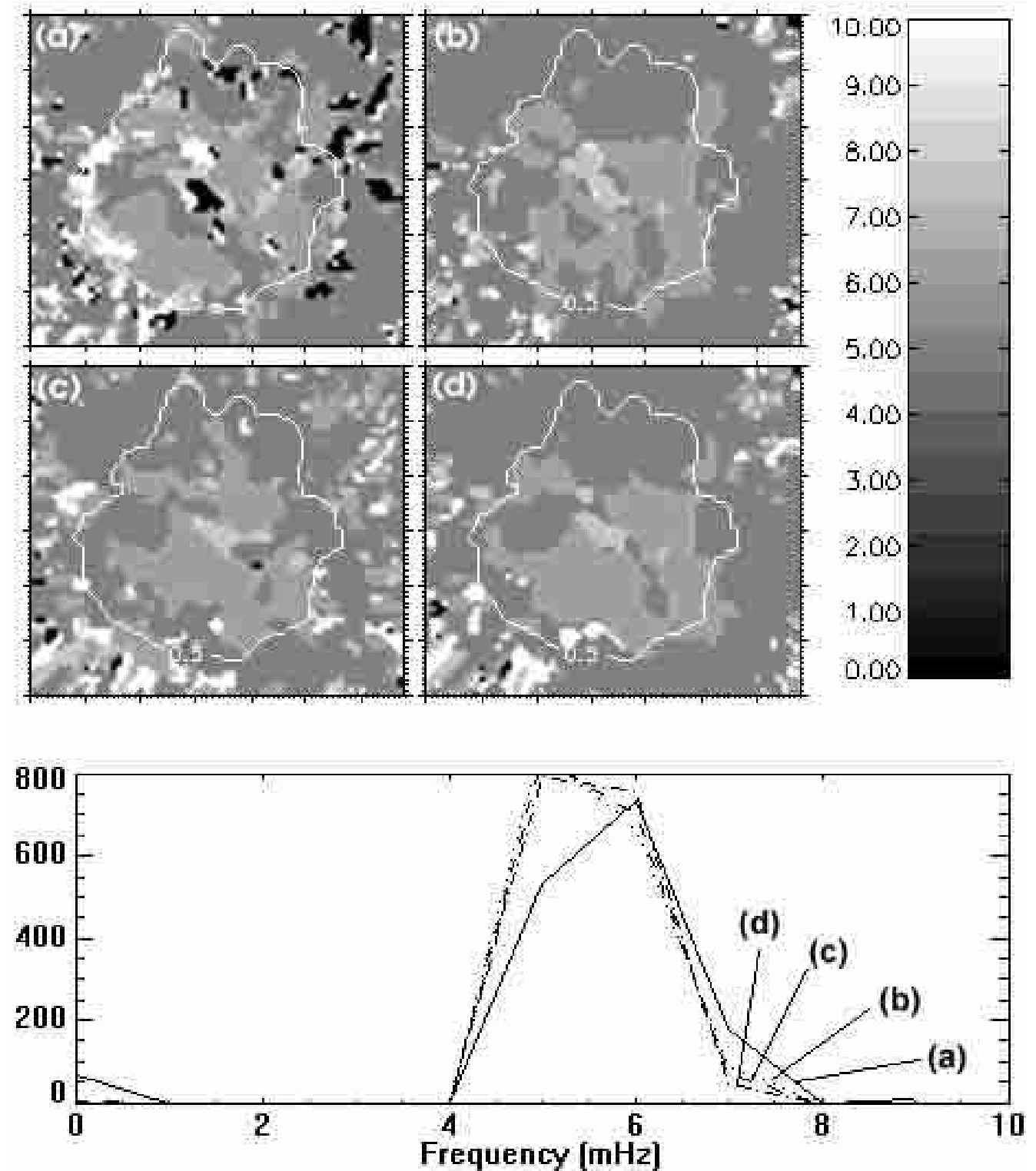

Fig. 8. Maps of "frequencies of maximum spectral power" computed from a) H $\alpha$ center b) $\mathrm{H} \alpha-0.5 \AA \mathbf{c}) \mathrm{H} \alpha+0.5 \AA$ and d) $\mathrm{H} \alpha \pm 0.5$ Doppler signal images, using a 256-point FFT (see Fig. 1). Contours delineate the umbra; the histogram below the map shows the frequency distribution of the pixels related to the umbra for the four wavelengths. The intensity bar on the right of the image, scales brightness to frequency values in $\mathrm{mHz}$.

Table 1. Observations.

\begin{tabular}{llcc}
\hline \hline Sequence & Wavelengths & Duration & Time Internal \\
\hline \hline $\mathrm{A}$ & $\mathrm{H} \alpha$ center and $\pm 0.5 \AA$ & 53 min & $12 \mathrm{~s}$ \\
$\mathrm{~B}$ & $\mathrm{H} \alpha$ center, $\pm 0.35, \pm 0.75$, Fe I $(5576.099) \pm 0.12 \AA$ & 116 min & $28 \mathrm{~s}$ \\
$\mathrm{C}$ & $\mathrm{H} \alpha \pm 0, \pm 0.35, \pm 0.5, \pm 0.75, \pm 1.0 \AA$. & a few minutes & $36 \mathrm{~s}$ \\
\hline \hline
\end{tabular}

The time interval is referred to the time between successive images of the same wavelength.

$\mathrm{H} \alpha \pm 0.75 \AA$. "Space/time slice images" computed using method II gives the opportunity to trace them in $\mathrm{H} \alpha-0.5 \AA$ and thus compare their propagation characteristics in different heights of the solar atmosphere.
Figures 2a and 2b show "space/time slice images" in $\mathrm{H} \alpha$ center and $\mathrm{H} \alpha-0.35 \AA$ respectively computed using the B sequence of observations. The "space/time slice images" are computed along exactly the same cut. The maximum power frequency along the "cut" 
[a]

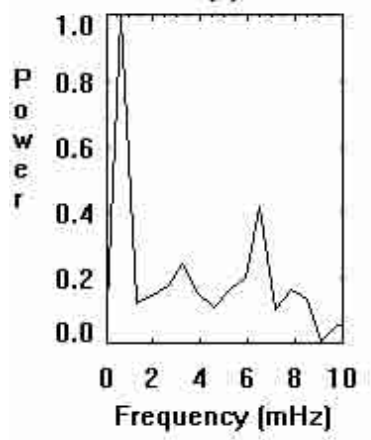

[c]

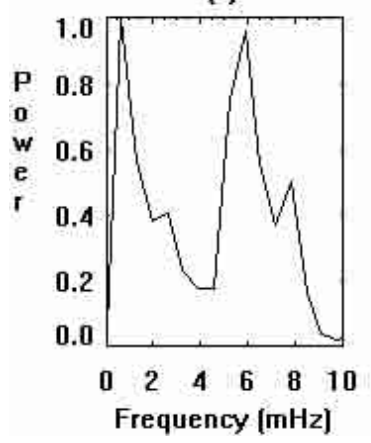

[b]

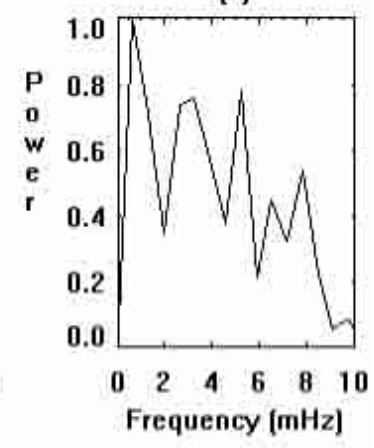

[d]

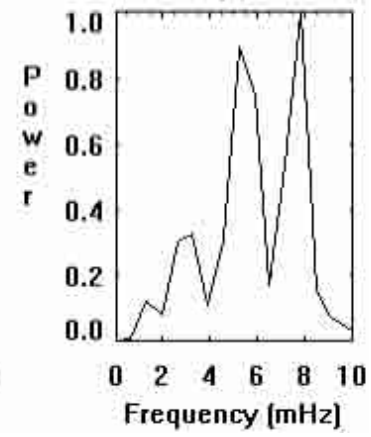

Fig. 9. Mean power spectra over the umbra in a) $\mathrm{H} \alpha$ center b) $\mathrm{H} \alpha-0.5 \AA$ c) $\mathrm{H} \alpha+0.5 \AA$ and d) $\mathrm{H} \alpha \pm 0.5$ Doppler velocity images. In order to compute the spectra we averaged the intensity over the penumbra and then computed the power spectrum for each wavelength.

in the area of the umbra is about $6.5 \mathrm{mHz}$ and in the penumbra $3.25 \mathrm{mHz}$ in both wavelengths. In order to determine possible lags in the propagation of the waves, we studied the correlation coefficient $C(\Delta t)$ as a function of time:

$C(\Delta t)=\frac{\Sigma\left(I_{\mathrm{H} \alpha 0}(t) I_{\mathrm{H} \alpha-0.3}(t+\Delta t)\right)}{\sqrt{\Sigma\left(I_{\mathrm{H} \alpha 0}^{2}(t)\right) \Sigma\left(I_{\mathrm{H} \alpha-0.3}^{2}(t)\right)}} \cdot$

A similar technique was adopted from Bocchialini \& Baudin (1995) in order to study the lags between signals coming from quiet sun oscillations in different layers of the solar atmosphere. We computed the correlation coefficient for each point of every row of a "space/time slice image", shifting the second image over the first one from minus to plus half the duration of the time series, producing in this way a "correlogram".

Figure 2c shows the "autocorrelogram" of the $\mathrm{H} \alpha$ center image and Fig 2d the "correlogram" between the $\mathrm{H} \alpha$ center and the $\mathrm{H} \alpha-0.35 \AA$. The study of the "correlogram" shows that there is a time difference in the propagation of RPWs of the order of $24 \mathrm{~s}$; a lag of about $10 \mathrm{~s}$ is also obvious in the umbral oscillations which, however, can be considered ambiguous. In order to evaluate if this lag is real or is related to an intensity-velocity phase shift, we computed the "correlogram" between the $\mathrm{H} \alpha-0.35 \AA$ line and (a) the $\mathrm{H} \alpha+0.35 \AA$ line, (b) the Doppler velocity. In both cases we found a time lag in the propagation of

RPWs, corresponding to a phase shift of $180^{\circ}$ (Fig. $3 \mathrm{~b}$ shows the "correlogram" between the $\mathrm{H} \alpha-0.35 \AA$ and the Doppler image). Since the Doppler velocity is computed by red blue wing subtraction, the red wing and the Doppler velocity has phase shift $0^{\circ}$ (we verified this result). The phase shift between the two wings corresponds to a pure Doppler velocity shift of the line. Figure $3 \mathrm{c}$ shows the "correlogram" between the $\mathrm{H} \alpha$ center image and the Doppler velocity. The correlogram shows a time difference in the propagation of the waves corresponding to a phase shift of $180^{\circ}$ plus the $24 \mathrm{~s}$ lag. This strongly indicates that the time lag in the propagation of the RPWs is real.

Figures $4 \mathrm{a}$ and $4 \mathrm{~b}$ show "space/time slice images" in $\mathrm{H} \alpha$ center and $\mathrm{H} \alpha-0.5 \AA$ respectively, computed using the A sequence of observations. As in the case of the B sequence the maximum power frequency along the "cut" in the area of the umbra is about $6.5 \mathrm{mHz}$ and in the penumbra $3.25 \mathrm{mHz}$ in both wavelengths. Figures $4 \mathrm{c}$ and $4 \mathrm{~d}$ show the autocorrelation for the $\mathrm{H} \alpha$ and the correlation between the two wavelengths respectively. From the "correlogram" we observe that there is a time difference of the order of $44 \mathrm{~s}$ for the RPWs and about $20 \mathrm{~s}$ for the oscillations.

From both the above comparisons we conclude that there is a systematic lag in the propagation of RPWs and the variation of umbral oscillations as they appear in different depths of the solar chromosphere.

Musman et al. (1976) found in Fe I 5576 isolated wave packets propagating outward across the penumbra. The waves were intermittent and had higher horizontal phase velocity by a factor of 2 or 3 , compared to the penumbral ones. In Paper II we found slow propagating photospheric waves probably related to the subphotospheric layer large-scale convection (see introduction). We continued our search in order to find the waves of Musman et al. (1976). The enhancement method incorporating the subtraction of the "running local average" results in washing out waves with periods larger than the integration time. Thus we experimented with increasing the integration time in successive steps in order to find waves with higher periods than the chromospheric ones $(5 \mathrm{~min})$. Figures $5 \mathrm{a}$ and $5 \mathrm{~b}$ show photospheric waves apparent in $\mathrm{H} \alpha-0.75 \AA$ and $\mathrm{FeI}(5576.099)-0.12 \AA$. The waves appear to begin not from the outer umbral boundary, but at approximately 0.7 of the distance between the umbra and the penumbra boundaries; the dividing line between inward and outward slow photospheric waves (see Paper II). The waves clearly propagate beyond the penumbra in the area around the sunspot, they are weak and quasi periodic. Their propagation velocity is around $3-4 \mathrm{~km} \mathrm{~s}^{-1}$ in the penumbra reduced to about $2 \mathrm{~km} \mathrm{~s}^{-1}$ in the area around the sunspot. They seem to be related to the same mechanism as the slow photospheric waves. There is a negative lag in their propagation between $\mathrm{H} \alpha-0.75 \AA$ and Fe I(5576.099)-0.12 A. As it can be seen from Figs. 6 and 7 the oscillation frequency in the area of the waves is of the order of $1-2 \mathrm{mHz}$, however it is very difficult to verify that it is related exclusively to the waves. Our results are consistent with those of Sigwarth \& Mattig (1997) who 

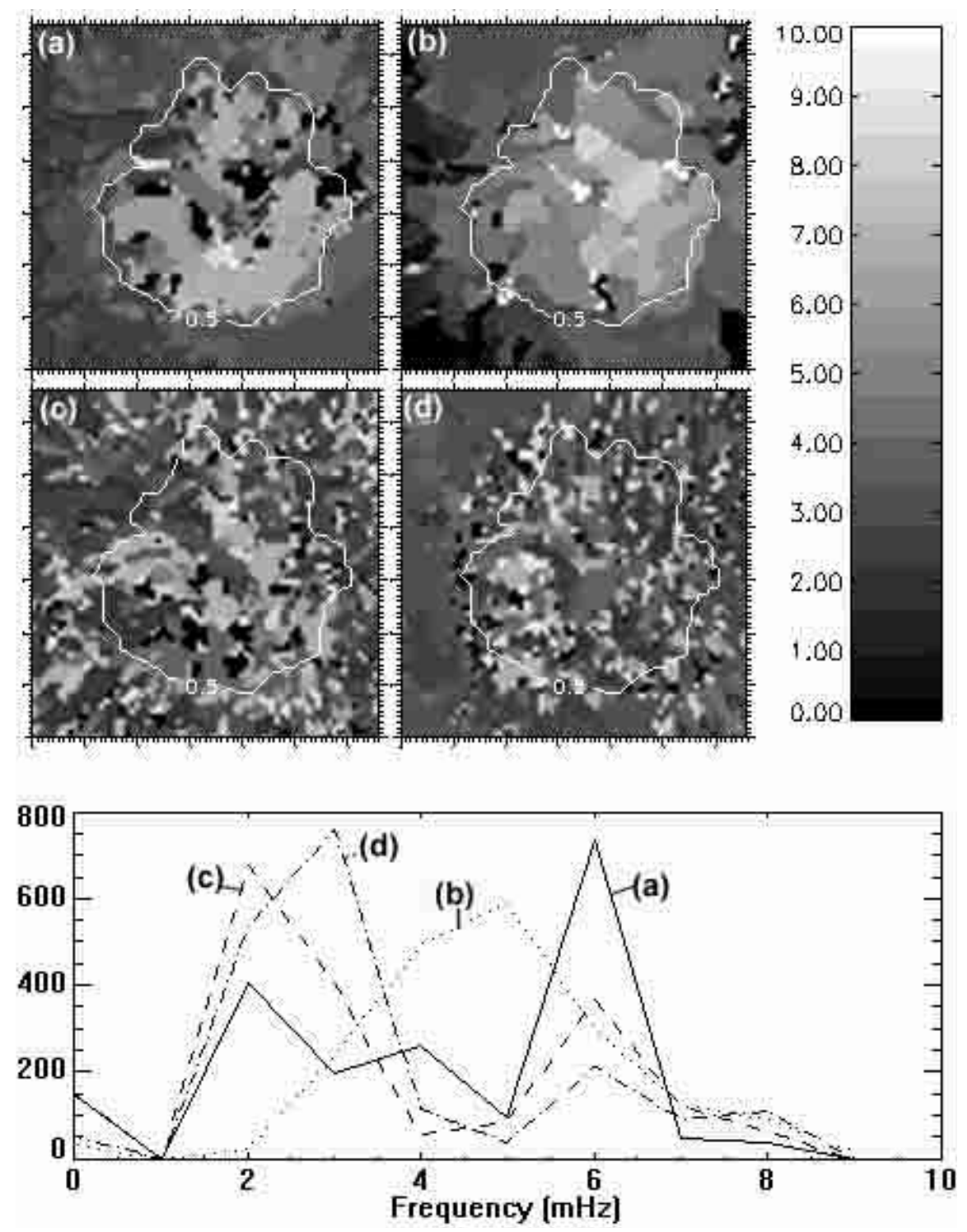

Fig. 10. Maps of "frequencies of maximum spectral power" computed from a) H $\alpha$ center and Doppler signal images in b) $\mathrm{H} \alpha \pm 0.35 \AA$ c) $\mathrm{H} \alpha \pm 0.75 \AA$ and d) Fe I $\pm 0.12 \AA$ using a 128-point FFT (see Fig. 1). Contours delineate the umbra; the histogram below the map shows the frequency distribution of the pixels related to the umbra for the four wavelengths. The intensity bar on the right of the image, scales brightness to frequency values in $\mathrm{mHz}$.

found long period fluctuations (about $30 \mathrm{~min}$ ) at the outer penumbra border with small phase speed of the order of 2 to $3 \mathrm{~km} \mathrm{~s}^{-1}$.

Distinct from the running waves, $3 \mathrm{~min}$ (about $6.25 \mathrm{mHz}$ ) standing oscillations are obvious in the inner penumbral area in $\mathrm{H} \alpha-0.75 \AA$ from both the "space/time slice images" and the "frequency/space maps" (Fig. 6). These results are further verified from the computation of the mean power spectrum of the penumbra (not shown here) that indicates significant power around $6.25 \mathrm{mHz}$. The oscillations are absent (or have much less power) in Fe I (5576.099)-0.12 ^ (Fig. 7). We recall that in Paper I we found that standing oscillations are not restricted in the umbral area but intrude well into the penumbra; they were more obvious at $\mathrm{H} \alpha+0.35 \AA$. In both Figs. 6 and 7 we observe high power near the Nyquist frequency $(15 \mathrm{mHz})$ that we do not consider real.

\subsection{Umbral oscillations in different layers of the solar atmosphere}

In order to study the temporal behavior of umbral oscillations in different layers of the solar atmosphere we computed "maximum power frequency maps" and mean power spectra in all available wavelengths (see Table I). The mean power spectra were computed by averaging the intensity over the umbra and then computing the power spectrum for each wavelength. Power spectra at different spatial points verify the findings of Kentischer \& Mattig (1995), that every spectrum contains a variety of different 
[a]

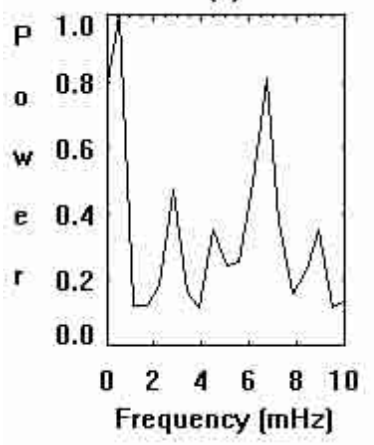

[c]

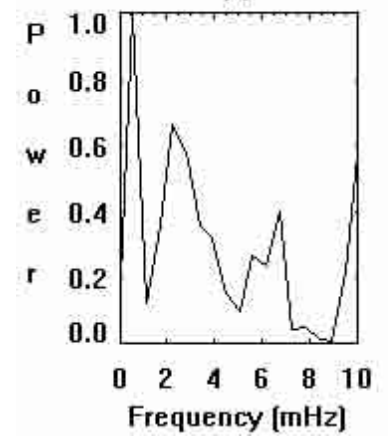

[b]

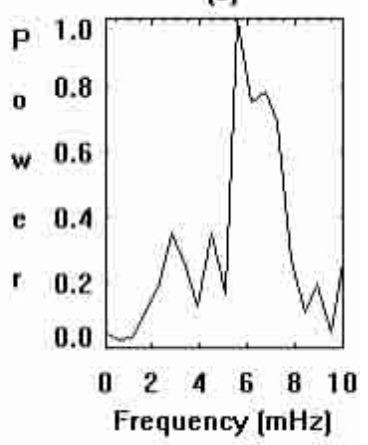

[d]

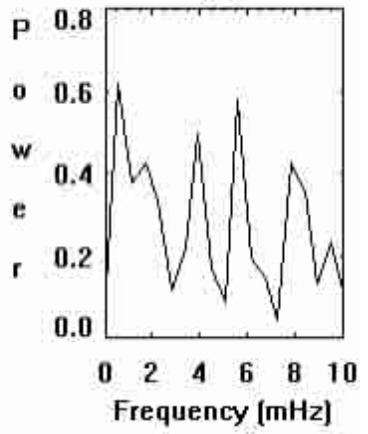

Fig. 11. Mean power spectra over the umbra in a) $\mathrm{H} \alpha$ center and Doppler signal images in b) $\mathrm{H} \alpha \pm 0.35 \AA$ c) $\mathrm{H} \alpha \pm 0.75 \AA$ and d) Fe $\mathrm{I} \pm 0.12 \AA$. In order to compute the spectra we averaged the intensity over the penumbra and then computed the power spectrum for each wavelength.

oscillation frequencies. Figure 8 shows "maximum power frequency maps" at $\mathrm{H} \alpha$ center (a), $\mathrm{H} \alpha-0.5 \AA$ (b), $\mathrm{H} \alpha+0.5 \AA$ (c) and $\mathrm{H} \alpha \pm 0.5$ (d) Doppler signal images, using the A sequence of observations. Figure 9 shows the mean power spectra for the same wavelengths. The results are partially influenced by the appearance of a light bridge at the left part of the spot, especially obvious at the $\mathrm{H} \alpha$ center image. We observe that the dominant oscillations at the core of the umbra are near $6 \mathrm{mHz}$ and there is a coherent behavior in all wavelengths. In intensity images we observe a concentration of power at about $0.5 \mathrm{mHz}$ related with the long term evolution of the sunspot. We should note that in the computation of the "maximum power frequency" maps, if the maximum power frequency was near $0.5 \mathrm{mHz}$, we aborted it and select the second maximum power frequency as the most characteristic oscillating frequency. The mean power spectrum at $\mathrm{H} \alpha$ center shows a major maximum at about $6.5 \mathrm{mHz}$, a secondary one at about $3.3 \mathrm{mHz}$ and finally a smaller one at about $8 \mathrm{mHz}$. As it is obvious from Fig. 8a, the concentration at $3.3 \mathrm{mHz}$ is mainly related to points near the boundaries of the umbra and not to those of the core. Further, the power near $8 \mathrm{mHz}$, which is more obvious in the other wavelengths and especially in the Doppler velocity at $\mathrm{H} \alpha \pm 0.5 \AA$ seems to be related to mass motions associated with the light bridge. The mean power spectrum of the Doppler velocity at $\mathrm{H} \alpha \pm 0.5$ shows a sim-

ilar behavior, except for the higher concentration of power at $8 \mathrm{mHz}$, already mentioned. The most interesting feature observed in the two opposite wings is that the power is concentrated in slightly displaced frequencies, a behavior also observed at $\mathrm{H} \alpha \pm 0.75$. If this is real, a probable explanation is that two different oscillations are propagating in different directions (upwards and downwards).

Figure 10 shows "maximum power frequency maps" at $\mathrm{H} \alpha$ center (a), and Doppler signal images at $\mathrm{H} \alpha \pm$ $0.35 \AA$ (b), $\mathrm{H} \alpha \pm 0.75 \AA$ (c), and FeI $\pm 0.12 \AA$ (d). Figure 11 shows the corresponding mean power spectra; the maps and the spectra are computed from the B sequence of observations. We observe that the maps computed from the Doppler images at $\mathrm{H} \alpha \pm 0.75 \AA$ and at Fe $\mathrm{I} \pm 0.12 \AA$ are much more noisy.

According to Lites (1986) the multiple peaks frequently observed in average umbral power spectra are due to the presence of cospatial modes with different frequencies. Kentischer \& Mattig (1995) found that the shape of each spectrum becomes completely different between two umbral points, separated by more than $3-5$ arcsec. The "maximum power frequency map" computed from both the $\mathrm{H} \alpha-0.35 \AA$ intensity and $\mathrm{H} \alpha \pm 0.35 \AA$ Doppler image shows a similar behavior (see Figs. 1 and 10b). Umbra appear to be composed of elements oscillating with slightly different frequencies. This is also obvious from the frequency distribution of the pixels related to the umbra of the Doppler image at $\mathrm{H} \alpha \pm 0.35 \AA$ that shows a large dispersion around $5 \mathrm{mHz}$ (Fig. 11).

The oscillating mode around $6 \mathrm{mHz}$ is present in every atmospheric layer; the number of points having more power at this frequency decreases as we move to lower layers. From both Figs. 10 and 11 we see that there is a concentration of power around $2 \mathrm{mHz}$ at photospheric layers $(\mathrm{H} \alpha \pm 0.75 \AA$ and at $\mathrm{Fe} \mathrm{I} \pm 0.12 \AA)$. This is in agreement with Lites \& Thomas (1985) who associated frequencies around $2 \mathrm{mHz}$ with the presence of gravity waves.

\subsection{Association between umbral oscillations and RPWs}

In Paper I, from "space/time slice images", we found that RPWs are not the continuation of umbral oscillations into the penumbra and that the period of RPWs is about twice that of umbral oscillations. Moreover, near the umbra- penumbra boundary, the two oscillating modes are clearly distinguished and there is not a smooth transition from one to the other. Figure 1 verifies that the dominant frequency of umbral oscillations is about twice that of RPWs. In order to further investigate their relation, we computed "correlograms", similar to that of Sect. 3.1. In this case however, we correlated the average variation of umbral or penumbral oscillations with every row of the "space/time slice" image. The variation of umbral oscillations was computed averaging the points along the space dimension of the "space/time slice" image corresponding to the umbra. The variation of penumbral oscillations 
was computed averaging 10 penumbral points at a distance of about $1 / 3$ between the umbra and the penumbra boundaries. They were selected this way so as not to be influenced by umbral oscillations. Finally we computed the correlation coefficient for each point of every row of the "space/time slice image", shifting the variation of um$\mathrm{bral} /$ penumbral oscillations over each row, from minus to plus half the duration of the time series. In the following we assume that the variations in the area of the umbra are related with the umbral oscillations and the variations in the penumbra with the RPWs. Figure 12 shows an $\mathrm{H} \alpha$ center "space/time slice image" (a), the correlation with the variation of the umbral oscillations (b), as well as the correlation with the variation of the penumbral oscillations (c). Figure 12c makes obvious the propagating nature of RPWs. It is further clear that there is not a significant correlation between the variation of umbral oscillations and that of RPWs in $\mathrm{H} \alpha$ center. Figure 13 shows the correlation of the variation of umbral oscillations computed from Doppler images at $\mathrm{H} \alpha \pm 0.35 \AA$ (a), $\mathrm{H} \alpha \pm 0.75 \AA$ (b), and Fe I $\pm 0.12 \AA$ (c), with a "space/time slice image" at $\mathrm{H} \alpha$ center. From Fig. 13a we observe that there is a significant correlation between the variation of umbral oscillations in $\mathrm{H} \alpha \pm 0.35 \AA$ Doppler images and in $\mathrm{H} \alpha$ center but not between the variation of umbral oscillations and that of RPWs. The lag in the variation of umbral oscillations between the $\mathrm{H} \alpha$ center and $\mathrm{H} \alpha \pm 0.35 \AA$ is confirmed. The variation of umbral oscillations in $\mathrm{H} \alpha \pm 0.75 \AA$ Doppler images shows a moderate correlation with both the variation of umbral oscillations and that of RPWs in $\mathrm{H} \alpha$ center (Fig. 13b). Finally, the variation of umbral oscillations in Fe I $\pm 0.12 \AA$ Doppler images shows a significant correlation with that of RPWs in $\mathrm{H} \alpha$ center (taking into consideration the lag in the propagation of the oscillations).

\section{Discussion and conclusions}

Lites \& Thomas (1985) from simultaneous observations in the photosphere and the chromosphere found that the 3-min oscillations have the character of a coherent vertically standing wave in the photosphere. The 5-min oscillations showed a small, positive phase difference between the low photosphere and the low chromosphere. Kentischer \& Mattig (1995) studied oscillations above sunspot umbrae at various atmospheric heights. They found two distinct distributions, one at about $6 \mathrm{mHz}$ that had the characteristics of an upward propagating wave travelling with the sound speed $\left(7 \mathrm{~km} \mathrm{~s}^{-1}\right)$ and a second distribution at $7.5 \mathrm{mHz}$ that had the characteristics of standing waves. Our results show that there is a lag in the signal of umbral oscillations with a frequency around $6 \mathrm{mHz}$, from the lower to the upper chromospheric layers.

Sigwarth \& Mattig (1997), from the analysis of oscillations at various atmospheric heights, found that sunspot penumbrae show 5-min oscillations from the inner to the outer penumbra boundary up to the lower chromospheric layers, but are not detectable in the layers below the temperature minimum. In the upper chromospheric layers they found a significant change in the frequencies from the central penumbra ( 4 to $3 \mathrm{mHz}$ ) to the outer penumbra border $(2 \mathrm{mHz})$. Finally they found upward propagating waves in the inner penumbra. Lites (1988) found that at frequencies near $3.3 \mathrm{mHz}$, penumbral oscillations in the Fe I $5344 \AA$ line lead those in the Ca II $8498 \AA$ line by $30^{\circ}-40^{\circ}$. Our results show that RPWs, having the same propagation characteristics and frequency as the $\mathrm{H} \alpha$ center ones, can be identified in the solar chromosphere at least up to the formation height of the $\mathrm{H} \alpha \pm 0.5 \AA$ line. The RPWs are not identifiable in the $\mathrm{H} \alpha \pm 0.75 \AA$ or the Fe $\mathrm{I} \pm 0.12 \AA$ intensity or Doppler images. We found a time lag between the waves in the blue and the red wing of $\mathrm{H} \alpha$ corresponding to a phase shift of $180^{\circ}$. This verifies that the disturbance related to RPWs is periodic transverse mass motions (see Discussion and conclusions in Paper I). The full thickness of the penumbral chromosphere moves up and down in the waves but not simultaneously. There is a systematic lag in the propagation of RPWs from the lower to the upper chromospheric layers. Finally we found 3-min penumbral oscillations apparent in the inner penumbra at lower chromospheric layers (far $\mathrm{H} \alpha$ wings). This verifies our previous results that more than one oscillating modes coexist in the penumbra.

Lites et al. (1982) studied sunspot oscillations in the chromospheric line Ca II $8542 \AA$. They found disturbances in the umbra and the penumbra with transverse propagation velocities of the order of $60-70 \mathrm{~km} \mathrm{~s}^{-1}$ and 20-35 $\mathrm{km} \mathrm{s}^{-1}$. They proposed that there is a relationship between the propagating umbral disturbances and the running penumbral waves. Kentischer \& Mattig (1995), found that proper motions were apparent in the oscillating elements of the umbra in their $\mathrm{H} \alpha$ and $\mathrm{Ca}$ II observations. The direction of the motions was mainly radial with velocities of the order of $23 \mathrm{~km} \mathrm{~s}^{-1}$ in Ca II and $19 \mathrm{~km} \mathrm{~s}^{-1}$ in $\mathrm{H} \alpha$. Their explanation was that the phenomenon is due to the slightly inclined magnetic field lines, which lead to a spreading out of the previously more compact oscillations. If the magnetic field is well structured, this may lead also to a concentric pattern, similar to concentric waves on water surfaces (see Fig. 3 in Paper I, where three such oscillating elements are obvious). Similar patterns were observed by Alissandrakis et al. (1992). Lites (1988) from Doppler movies of sunspots in Fe I observed a continuity of the disturbances across the umbral-penumbral boundaries. He proposed that the disturbances may be due to a common physical mechanism operating both in the umbra and the inner penumbra. Our results show a significant correlation of the photospheric umbral oscillations with the running penumbral waves observed in the chromosphere. Although it should be critically examined if this relationship is real or chance, the fact that the full thickness of the penumbral chromosphere moves up and down in the waves and the systematic lag in the propagation of RPWs from the lower to the upper chromospheric layers enhance the hypothesis of the relation of the two phenomena. 


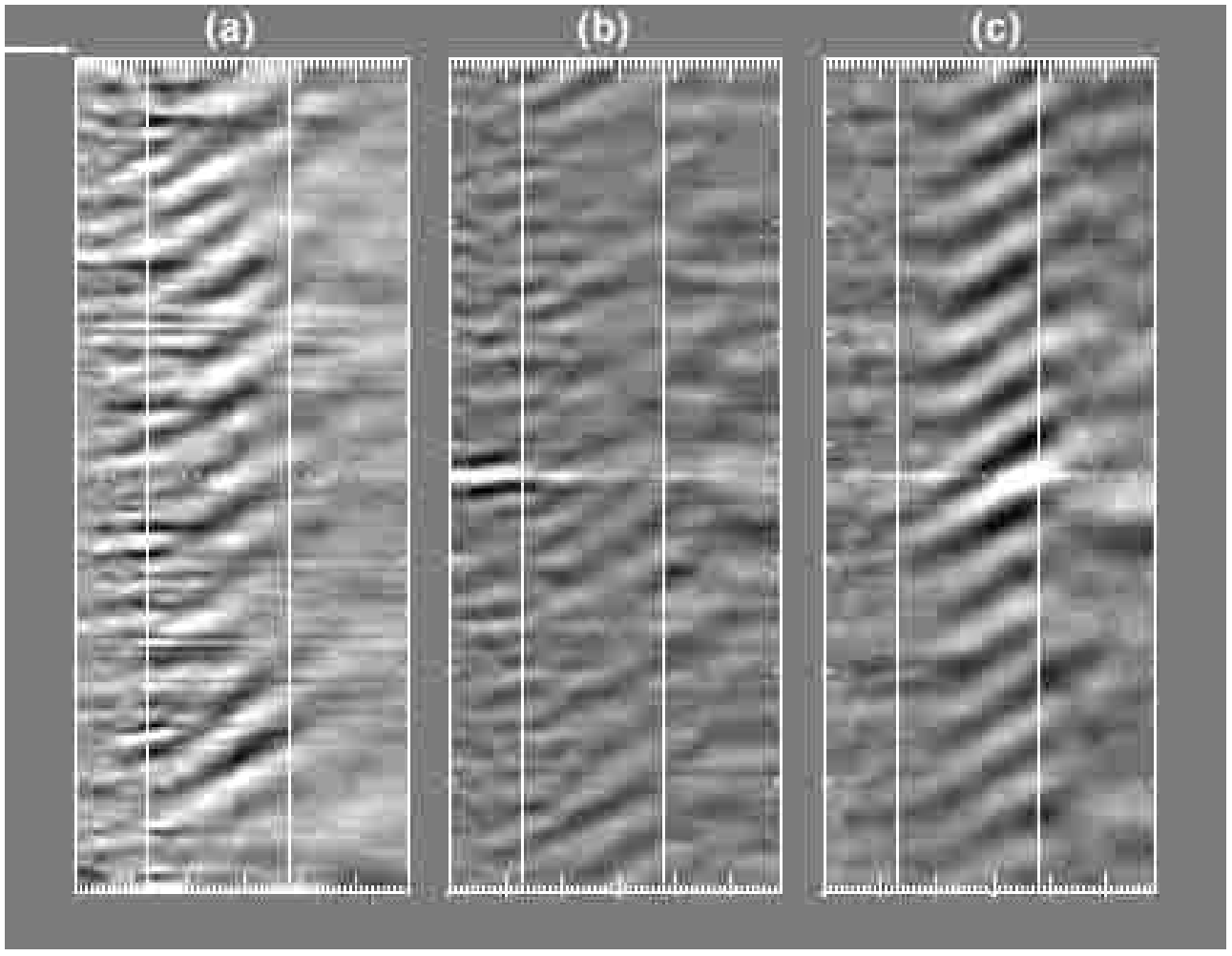

Fig. 12. Correlation of the average variation of umbral oscillations/RPWs with a "space/time slice image". In a) we show the "space/time slice" image in $\mathrm{H} \alpha$ center, in b) the "correlogram" of the average variation of umbral oscillations with the "space/time slice" image and in c) the "correlogram" of the average variation of RPWs with the "space/time slice" image. See text for further explanations. Tick marks in the $x$-axis correspond to 0.26 "and tick marks in the $y$-axis to $560 \mathrm{~s}$.

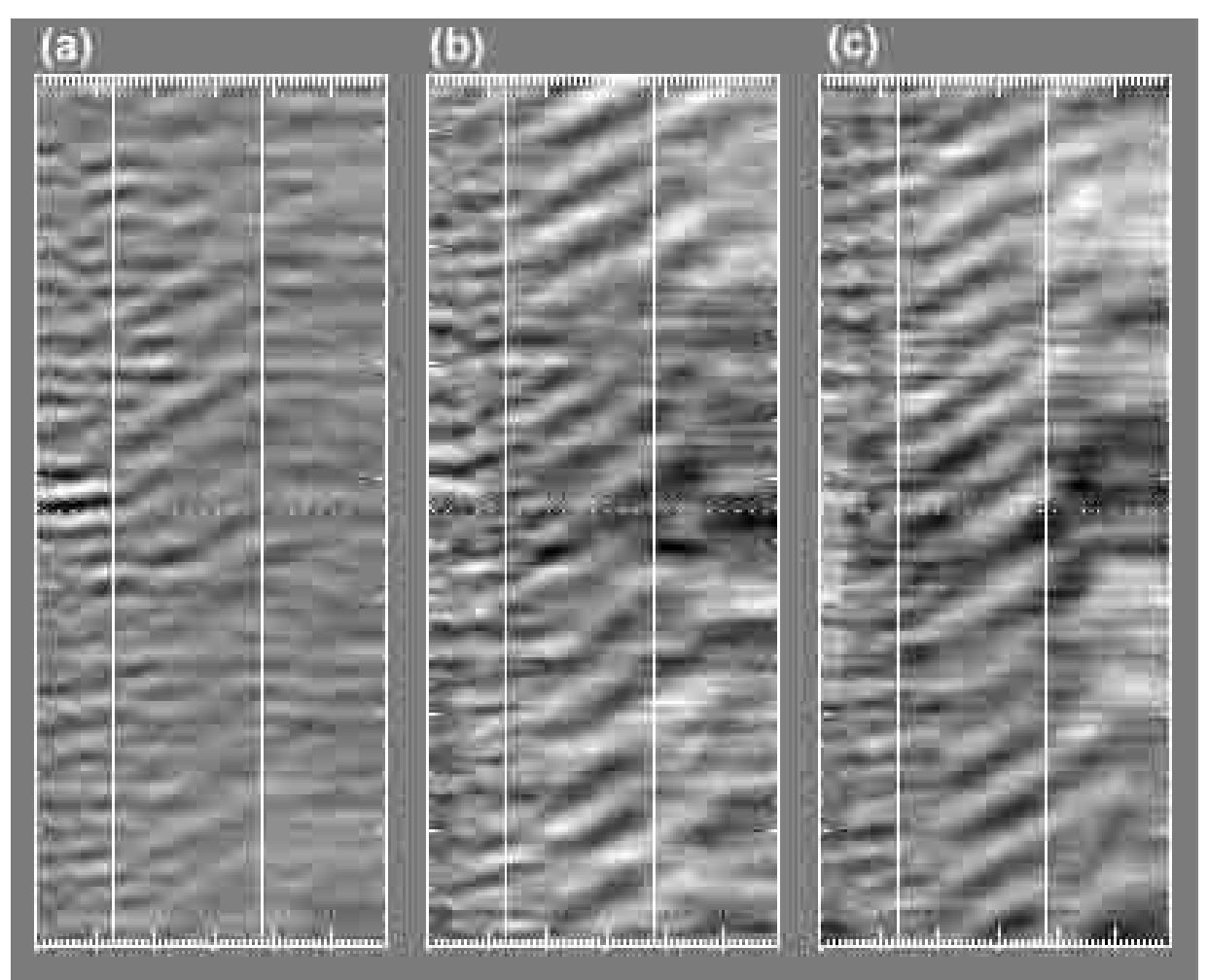

Fig. 13. Correlation of the variation of umbral oscillations computed from Doppler images at $\mathrm{H} \alpha \pm 0.35 \AA$ a), H $\alpha \pm 0.75 \AA$ b), and Fe $\mathrm{I} \pm 0.12 \AA \mathbf{c}$ ), with a "space/time slice image" at $\mathrm{H} \alpha$ center (see Fig. 12a). Tick marks in the $x$-axis correspond to $0.26^{\prime \prime}$ and tick marks in the $y$-axis to $140 \mathrm{~s}$. Compare the "correlograms" with Fig. 12c. 
The observations of Lites (1988) further enhance this scenario since the Fe I line is formed near the temperature minimum. However, in the case of the chromospheric umbral oscillations we consider more likely the scenario proposed by Kentischer \& Mattig (1995) that creates the false impression of a continuity between the umbral disturbances and the RPWs.

Rimmele (1994) and Shine et al. (1994) found that Evershed velocities are non-stationary. They observed velocity packets propagating radially outward, towards the penumbral boundary and into the adjacent quiet photosphere. The average propagation speed was about $3.2 \mathrm{~km} \mathrm{~s}^{-1}$, subsequent velocity packets were separated in space by $2000-3000 \mathrm{~km}$ and the pattern repeated in a quasi periodic way on a time scale of the order of $15 \mathrm{~min}$. The velocity packets were travelling in Evershed channels and they did not find a significant phase relation linking velocity packets in adjacent Evershed channels. Rimmele (1994) noticed that the period matches the long term oscillations observed in the penumbra. The velocity packets described above present significant similarities with the photospheric ones we presented in Sect. 3.1. However, our waves present a coherence along arcs of the order of $30^{\circ}$. If it is shown that the two phenomena are related, then their association with the Evershed flow should be critically examined since the waves can also be observed at $\mathrm{H} \alpha \pm 0.75 \AA$ (Fig. 5), where the Evershed flow is reversed.

Based on the previous results, as well as the results of the works referred to above, we propose the following model concerning the umbral oscillations and running penumbral waves:

(i) Chromospheric Umbral Oscillations with a frequency of about $6.5 \mathrm{mHz}$ are related to oscillations at the lower layers of the chromosphere.

(ii) RPWs propagating with a constant velocity around $13 \mathrm{~km} \mathrm{~s}^{-1}$ and having a frequency around $3.3 \mathrm{mHz}$ are excited from photospheric umbral oscillations. The waves are initiated at low chromospheric levels and the disturbance propagates upwards.

(iii) The above waves are not detectable in the photosphere where other kinds, of slow waves exist. The nature of the photospheric waves needs further research.

(iv) Three minute standing oscillations are apparent in the inner penumbra. The oscillations are more obvious in the $\mathrm{H} \alpha+0.35 \AA$ and far $\mathrm{H} \alpha$ wings where the RPWs are respectively weak or not apparent.
Further investigation of the nature of oscillations and waves related to sunspots requires more sophisticated observations of higher sensitivity, simultaneous study of related phenomena and further theoretical progress.

Acknowledgements. We would like to thank Dr. R. N. Smartt, the T.A.C. of NSO/SP and the staff of the Sacramento Peak Observatory, especially the Observers team of the DST, for their warm hospitality and their help with the observations. Further, we would like to thank the referee (Dr. H. Wöhl) for his suggestions that helped improve the text. This work was partially supported by NSF grant ATM-9726147.

\section{References}

Alissandrakis, C. E., Georgakilas, A. A., \& Dialetis, D. 1992, Solar Phys., 138, 93

Bocchialini, K., \& Baudin, F. 1995, A\&A, 299, 893

Bogdan, T. 2000, Solar Phys., 192, 373

Brisken, W. F., \& Zirin, H. 1997, ApJ, 478, 814

Christopoulou, E. B., Georgakilas, A. A., \& Koutchmy, S. 2000, A\&A, 354, 305

Georgakilas, A. A., Christopoulou, E. B., \& Koutchmy, S. 2000, A\&A, 363, 306

Giovanelli, R. G. 1972, Solar Phys., 27, 71

Giovanelli, R. G. 1974, in IAU Symp. 56, Chromospheric Fine Structure, 137

Kentischer, T. J., \& Mattig, W. 1995, A\&A, 300, 539

Lites, B. W. 1986, ApJ, 301, 1005

Lites, B. W. 1988, ApJ, 334, 1054

Lites, B. W. 1992, in NATO ASIC Proc. 375, Sunspots. Theory and Observations, 261-302

Lites, B. W., \& Thomas, J. H. 1985, ApJ, 294, 682

Lites, B. W., White, O. R., \& Packman, D. 1982, ApJ, 253, 386

Moore, R. L. 1981, Space Sci. Rev., 28, 387

Musman, S., Nye, A. H., \& Thomas, J. H. 1976, ApJ, 206, L175

Rimmele, T. R. 1994, A\&A, 290, 972

Shine, R. A., Title, A. M., Tarbell, T. D., et al. 1994, ApJ, 430,413

Sigwarth, M., \& Mattig, W. 1997, A\&A, 324, 743

Soltau, D., Schröter, E. H., \& Wöhl, H. 1976, A\&A, 50, 367

Steinegger, M., Bonet, J. A., \& Vazquez, M. 1997, Solar Phys., 171,303

Zirin, H., \& Stein, A. 1972, ApJ, 178, L85 